\title{
Carnets
}

Revue électronique d'études françaises de l'APEF

Deuxième série - 8 | 2016

Du Français en cause aux causes du français

\section{Système éducatif portugais}

Enseignement du Français au Portugal

\section{Eulália Alexandre}

\section{(2) OpenEdition \\ Journals}

Édition électronique

URL : http://journals.openedition.org/carnets/1832

DOI : $10.4000 /$ carnets. 1832

ISSN : 1646-7698

Éditeur

APEF

Référence électronique

Eulália Alexandre, «Système éducatif portugais », Carnets [En ligne], Deuxième série - 8 | 2016, mis en ligne le 30 novembre 2016, consulté le 30 avril 2019. URL : http://journals.openedition.org/

carnets/1832 ; DOI : 10.4000/carnets.1832

Ce document a été généré automatiquement le 30 avril 2019.

\section{(c) (i) \&}

Carnets est mis à disposition selon les termes de la licence Creative Commons - Atribution - Pas d'utilisation commerciale 4.0 International. 


\title{
Système éducatif portugais
}

\author{
Enseignement du Français au Portugal
}

\section{Eulália Alexandre}

\section{Le système éducatif portugais - Principes et priorités}

1 Le système éducatif portugais se base sur un ensemble de principes précis, parmi lesquels on souligne :

- L'éducation préscolaire universelle pour des enfants à partir de 5 ans ;

- L'enseignement obligatoire pour tous les enfants et les jeunes pendant 12 ans - de 6 à 18 ans, ou jusqu'à la conclusion de l'enseignement secondaire ;

- Une offre éducative gratuite aux écoles maternelles et aux établissements scolaires publics ;

- La liberté de choix de l'établissement scolaire fréquenté - établissements publics et privés ;

- Un curriculum national et la gestion autonome des établissements scolaires.

2 En ce qui concerne les grandes priorités du système éducatif portugais, on peut référer les priorités suivantes :

- Offrir une formation de base commune, à travers l'acquisition de connaissances et le développement de capacités fondamentales ;

- Promouvoir une culture de rigueur et d'excellence dans l'éducation ;

- Améliorer la réussite scolaire et la qualité de l'éducation.

\section{Le système éducatif portugais - Structure}

3 Comme l'on a signalé, au Portugal l'enseignement est obligatoire pendant 12 ans, pour tous les élèves âgés de 6-18 ans ou jusqu'à ce qu'ils complètent l'enseignement secondaire.

4 L'enseignement obligatoire est fondamentalement divisé en deux étapes. La première étape est appelée ensino básico (enseignement élémentaire) et correspond à 
l'enseignement primaire et au collège. L'enseignement élémentaire dure 9 ans et il est divisé en 3 cycles de scolarité :

- Le $1^{\text {er }}$ cycle dure 4 ans - de la $1^{\text {ère }}$ année à la $4^{e}$ année - et il est destiné aux enfants âgés de 6 à 10 ans :

- Le $2^{\text {ème }}$ cycle dure 2 ans - de la $5^{\mathrm{e}}$ année à la $6^{\mathrm{e}}$ année - et il est destiné aux élèves âgés de 10 à 12 ans ;

- Le $3^{\text {ème }}$ cycle dure 3 ans - de la $7^{e}$ année à la $9^{e}$ année - et il est destiné aux élèves âgés de 12 à 15 ans.

5 La deuxième étape de l'enseignement obligatoire est appelé ensino secundário (enseignement secondaire) et correspond au lycée. L'enseignement secondaire dure 3 ans - de la $10^{\mathrm{e}}$ année à la $12^{\mathrm{e}}$ année - et il est destiné aux élèves âgés de 15 à 18 ans.

\section{Le système éducatif portugais - Cours}

6 Dans le système éducatif portugais, les élèves peuvent choisir parmi différentes modalités d'enseignement.

7 Ainsi, à l'enseignement élémentaire, il y a l'enseignement élémentaire général, les cours d'éducation artistique spécialisée, les cours de formation vocationnelle et l'éducation des adultes.

8 À l'enseignement secondaire, il y a les cours scientifiques et humanistiques, les cours à plans spécifiques, les cours artistiques spécialisés, les cours professionnels, les cours de formation vocationnelle et l'éducation des adultes.

\section{Le système éducatif portugais - Curriculum et matières}

9 Tel que l'on a indiqué, l'enseignement élémentaire général dure 9 ans et il est divisé en 3 cycles d'apprentissage : le $1^{\text {er }}$, le $2^{\text {ème }}$ et le $3^{\text {ème }}$ cycles.

$10 \mathrm{Au} 1^{\mathrm{er}}$ cycle, les enfants ont portugais (minimum de $7 \mathrm{~h} /$ semaine), mathématiques (minimum de $7 \mathrm{~h} /$ semaine), estudo do meio (histoire, géographie et sciences), expressions artistiques et physiques-motrices, étude surveillée et l'offre supplémentaire de l'établissement; ils peuvent avoir aussi des activités d'enrichissement du curriculum (AEC). À partir de 2015/2016, ils auront obligatoirement anglais comme langue étrangère (LE I).

$11 \mathrm{Au} 2^{\text {ème }}$ cycle, les élèves ont les matières suivantes : portugais (6h/semaine), anglais (LE I), histoire et géographie du Portugal, mathématiques (6h/semaine), sciences naturelles, éducation visuelle, éducation technologique, éducation musicale, éducation physique et formation religieuse (facultative).

$12 \mathrm{Au} 3^{\text {ème }}$ cycle, les élèves étudient portugais (5h/semaine), anglais et une deuxième langue étrangère (LE II), histoire, géographie, mathématiques (5h/semaine), sciences naturelles, physique-chimie, éducation visuelle, TICE, l'offre de l'établissement, éducation physique et formation religieuse (facultative).

13 Concernant l'enseignement secondaire et les cours scientifiques et humanistiques, la structure de ceux-ci se divise en deux composantes : la formation générale et la formation spécifique. 
14 À la formation générale, qui est commune à tous les cours scientifiques et humanistiques, les élèves étudient obligatoirement portugais, une langue étrangère I, II ou III (qui peut être l'allemand, l'espagnol, le français ou l'anglais), philosophie et éducation physique.

15 À la formation spécifique, dont l'objectif est de favoriser une préparation solide dans le domaine spécifique de chaque cours scientifique et humanistique, les élèves ont une matière triennale obligatoire $\left(10^{\mathrm{e}}, 11^{\mathrm{e}}\right.$ et $12^{\mathrm{e}}$ années) et ils choisissent: 2 matières biennales $\left(10^{\mathrm{e}}\right.$ et $11^{\mathrm{e}}$ années), directement liées à la nature scientifique du cours et 2 matières annuelles ( $12^{\mathrm{e}}$ année), une liée à la nature scientifique du cours et l'autre peut être liée à d'autres domaines scientifiques. La formation religieuse reste toujours optionnelle.

$16 \mathrm{Au}$ présent, l'accent est mis sur le renforcement de l'autonomie des établissements scolaires et sur la flexibilité accordée à l'organisation des activités scolaires.

17 Ainsi, il y a un temps maximum attribué à chaque cycle de l'enseignement élémentaire et à l'enseignement secondaire : l'horaire hebdomadaire peut aller jusqu'à 22,5-26h/semaine chaque année du $1^{\text {er }}$ cycle ; jusqu'à 22,5-23,2h/semaine au $2^{\text {ème }}$ cycle ; jusqu'à 25,5-26,2h/ semaine au $3^{\text {ème }}$ cycle ; et enfin jusqu'à 34-36h/semaine à l'enseignement secondaire.

Le Ministère de l'Éducation et de la Science (MEC) définit le temps minimum alloué à un domaine ou à une matière spécifique et le temps maximum par année ou par cycle, lesquels sont gérés de façon flexible par les établissements scolaires, en fonction de leurs besoins.

\section{Le système éducatif portugais - Évaluation et certification}

19 L'évaluation fait partie de la pratique éducative, en permettant une collecte systématique d'informations essentielles à la prise de décisions appropriées pour améliorer la qualité de l'apprentissage des élèves.

Par rapport à l'enseignement obligatoire, l'évaluation est organisée en trois modalités différentes : évaluation diagnostique, formative et sommative (interne et externe). D'un côté, les élèves de l'enseignement élémentaire (à l'exception du $1^{\text {er }}$ cycle) sont généralement évalués d'après une échelle d'évaluation sommative de 1-5, selon laquelle 3 correspond à la réussite; de l'autre côté, les élèves de l'enseignement secondaire sont évalués d'après une échelle d'évaluation sommative de $0-20$, selon laquelle 10 équivaut à la réussite.

Il y a des épreuves nationales (évaluation externe) de portugais et de mathématiques à la fin de chaque cycle de l'enseignement élémentaire, c'est-à-dire en $4^{\mathrm{e}}, 6^{\mathrm{e}}$ et $9^{\mathrm{e}}$ années.

À l'enseignement secondaire, l'élève doit obtenir une classification d'au moins $10 / 20$ pour toutes les matières du curriculum et, pour conclusion des études, il faut réaliser obligatoirement 4 examens nationaux des matières suivantes : portugais, la triennal et les deux biennales de la formation spécifique ; il y a aussi un examen national de philosophie, lequel est facultatif.

Pour terminer, il faut remarquer que les examens nationaux de l'enseignement secondaire peuvent se constituer comme épreuves d'accès à l'enseignement supérieur. 


\section{La situation de l'enseignement du français}

En ce qui concerne le français à l'enseignement élémentaire, il faut prendre en compte que la sensibilisation/initiation au français est prévue pour le $1^{\mathrm{er}}$ cycle, dans le cadre des activités d'enrichissement du curriculum (AEC), en fonction des projets éducatifs des établissements scolaires.

L'apprentissage du français comme deuxième langue étrangère (LE II) peut avoir lieu à partir du $3^{\text {ème }}$ cycle $\left(7^{\mathrm{e}}, 8^{\mathrm{e}}\right.$ et $9^{\mathrm{e}}$ années) et les élèves peuvent choisir entre l'allemand, l'espagnol et le français, en fonction de leurs intérêts et de l'offre des établissements scolaires. Les facteurs décisifs dans le choix du français semblent être encore la tradition et les affinités culturelles avec la France.

Par rapport au français à l'enseignement secondaire, les cours scientifiques et humanistiques garantissent, à la formation générale, une LE I, II ou III comme matière obligatoire ( $10^{\mathrm{e}}$ et $11^{\mathrm{e}}$ années); et, à la formation spécifique, une LE I, II ou III comme matière facultative ( $12^{\mathrm{e}}$ année).

Le français, niveau continuation ou initiation, se présente donc comme l'une des options, à côté d'autres langues comme l'allemand, l'espagnol et de l'anglais.

Il faut ajouter que le cours scientifique et humanistique de Langues et d'Humanités comprend également, dans la formation spécifique, la matière facultative de LE I, II ou III ( $10^{\mathrm{e}}-11^{\mathrm{e}}$ années et $12^{\mathrm{e}}$ année).

29 D'un point de vue général et relativement aux langues vivantes étrangères à l'enseignement secondaire, le MEC au Portugal reconnaît l'importance du plurilinguisme dans un monde globalisé et dans une Europe sans frontières, mais avec de différentes identités culturelles et linguistiques que nous devrions tous valoriser.

30 Il n'est, toutefois, possible ni souhaitable d'augmenter maintenant le nombre de matières obligatoires dans les cours scientifiques et humanistiques de l'enseignement secondaire.

31 Le MEC assure ainsi l'apprentissage obligatoire, pour tous les élèves de l'enseignement secondaire, d'une langue étrangère, niveau continuation ou initiation (LE I, II ou III). En outre, les élèves peuvent aussi continuer l'apprentissage d'une des langues étrangères apprises dans l'enseignement élémentaire, comme matière supplémentaire.

32 Selon les données indiquées par la Direction générale des Statistiques de l'Éducation et de la Science (DGEEC) au Portugal, le nombre d'élèves inscrits en français présente des oscillations depuis 2008/2009, soit à l'enseignement élémentaire ( $2^{\text {ème }}$ et $3^{\text {ème }}$ cycles), soit à l'enseignement secondaire :

\begin{tabular}{|l|l|l|l|}
\hline Année Scolaire & $\begin{array}{l}\text { N. bre élèves de français } \\
\text { à l'ens. élémentaire }\end{array}$ & $\begin{array}{l}\text { N. bre élèves de français } \\
\text { à l'ens. secondaire }\end{array}$ & N. bre total d'élèves \\
\hline $2008 / 2009$ & 272.796 & 30.947 & 303.743 \\
\hline $2009 / 2010$ & 256.410 & 29.649 & 286.059 \\
\hline $2010 / 2011$ & 247.285 & 32.266 & 279.551 \\
\hline $2011 / 2012$ & 239.392 & 29.110 & 268.502 \\
\hline
\end{tabular}




\begin{tabular}{|l|l|l|l|}
\hline $2012 / 2013$ & 239.891 & 26.331 & 266.222 \\
\hline
\end{tabular}

SOURCE : DGEEC (DONNÉES MOdIfIÉES EN JUIN 2015, EN IMPLIQUANT L'ENSEIgNEMENT PUBLIC ET L'ENSEIgNEMENT PRIVÉ)

On peut constater qu'il y a eu une diminution générale de l'apprentissage du français dans le système éducatif portugais : de 303.743 élèves en 2008/2009 à 266.222 élèves en 2012/2013. Cette baisse peut être liée au déclin général de la population scolaire; à l'augmentation de l'espagnol depuis son entrée dans le système éducatif portugais ; et, finalement, à une légère croissance de l'allemand, notée plus récemment.

À l'enseignement élémentaire, on a remarqué l'accroissement plus récent du français pendant l'année scolaire 2012/2013, avec 239.891 élèves.

À l'enseignement secondaire, le français a aussi augmenté pendant l'année scolaire 2010/2011, avec 32.266 élèves ; pourtant, les années suivantes, le nombre d'élèves inscrits en français a subi une autre diminution : 26.331 élèves en 2012/2013.

Malgré ces fluctuations, le français reste, en termes quantitatifs, la LE II la plus enseignée au Portugal, quand comparée avec l'allemand et l'espagnol.

En plus, il faut souligner que, d'après les données fournies par la DGEEC, le nombre total d'élèves inscrits en français reste élevé par rapport aux autres langues :

\begin{tabular}{|l|l|l|l|}
\hline Année Scolaire 2012/2013 & $\begin{array}{l}\text { Nb. d'élèves } \\
\text { à l'ens. élémentaire }\end{array}$ & $\begin{array}{l}\text { Nb. d'élèves } \\
\text { àens. secondaire }\end{array}$ & Nb. total d'élèves \\
\hline Allemand & 2.942 & 2.873 & 5.815 \\
\hline Espagnol & 92.033 & 28.848 & 120.881 \\
\hline Français & 239.891 & 26.331 & 266.222 \\
\hline
\end{tabular}

SOURCE : DGEEC (DONNÉES MOdIfIÉES EN JUIN 2015, EN IMPLIQUANT L'ENSEIgNEMENT PUBLIC ET L'ENSEIgNEMENT PRIVÉ)

En ce qui concerne les données officielles de l'année scolaire 2013/2014, selon la DGEEC, elles seront disponibles seulement à partir d'octobre 2015.

\section{Programmes et projets innovants en français}

Le Protocole de Coopération Éducative franco-portugais entre les ministères de l'Education du Portugal et de la France a été signé le 10 avril 2006, à Paris.

Réaffirmant l'intérêt pour améliorer l'apprentissage des langues étrangères et pour favoriser le multilinguisme, les signataires de ce protocole ont accordé de promouvoir l'offre en portugais et en français dans les systèmes éducatifs des deux pays, dans la perspective de développer une offre linguistique plurielle.

Dans le cadre de ce protocole, le Portugal a développé différents programmes et projets innovants dans les établissements scolaires de l'enseignement élémentaire et de l'enseignement secondaire, tels que les Assistants de Français, les Sections Européennes 
de Langue Française, les Séjours Professionnels et la certification DELF scolaire, dont on parlera ensuite.

\section{Programme des Assistants de Français}

Mis en œuvre depuis 1974, ce programme s'est développé jusqu'à présent sans aucune interruption et sur une base réciproque avec le Programme des Assistants de Portugais en France.

43 Ses objectifs principaux sont de sensibiliser les élèves à la connaissance de la langue française et des cultures francophones; de contribuer à l'amélioration des compétences linguistiques des élèves; et d'appuyer les enseignants de français à l'intérieur et à l'extérieur de la salle de cours.

44 Les destinataires de ce programme sont les établissements scolaires de l'enseignement élémentaire et de l'enseignement secondaire.

En ce qui concerne l'année scolaire 2014/2015, il y a 14 assistants français et 14 établissements scolaires d'affectation dans les cinq régions portugaises (4 au Nord, 3 au Centre, 3 à Lisbonne et la Vallée du Tage, 2 à l'Alentejo et 2 à l'Algarve).

\section{Projet des Sections Européennes de Langue Française (SELF)}

Mis en œuvre à partir de l'année scolaire 2006/2007, le projet des SELF au Portugal incluait au début 7 établissements scolaires et 227 élèves.

Ses objectifs principaux sont la valorisation de l'apprentissage de la langue française à travers un renforcement de $45 \mathrm{~min}$ de la charge horaire de français ; l'apprentissage de contenus d'une ou deux disciplines non linguistiques (DNL) en langue française (les classes EMILE/CLIL) ; le développement d'une identité européenne ; et la préparation des élèves aux défis de demain.

Les destinataires de ce projet sont les établissements scolaires de l'enseignement élémentaire et de l'enseignement secondaire.

49 Par rapport à l'année scolaire 2014/2015, on compte un total de 1339 élèves, 57 classes et 30 établissements scolaires dans les cinq régions portugaises (11 au Nord, 5 au Centre, 8 à Lisbonne et la Vallée du Tage, 3 à l'Alentejo et 3 à l'Algarve) - source: DGE/DGEstE (données modifiées en juin 2015, d'après le dernier comptage).

\section{Programme Séjours Professionnels}

50 Au Portugal, ce programme a été mis en œuvre à partir de 2009/2010, en ayant pour objectifs principaux de contribuer à l'amélioration linguistique, pédagogique et culturelle des enseignants (de langue ou de DNL) et de leur proposer un séjour de deux semaines pour l'observation et la participation à la vie scolaire des établissements scolaires portugais et français. 
51 Les bénéficiaires de ce programme bilatéral sont les enseignants français et portugais, de préférence ceux impliqués dans des projets de Sections Européennes en France et au Portugal.

Concernant l'année scolaire 2014/2015, 4 professeurs français seront accueillis dans des établissements portugais et 3 professeurs portugais seront reçus dans des établissements scolaires français sélectionnés au préalable.

\section{Certification DELF scolaire}

Cette certification a été mise en œuvre au Portugal depuis l'année scolaire 2007/2008. Selon les données de l'Institut Français du Portugal, en 2013/2014, le projet impliquait 28 centres de passation et 166 établissements scolaires publics. En outre, 1783 élèves portugais ont obtenu le diplôme et le taux de réussite a été de 98,6 \%

Premièrement, il faut souligner que l'évaluation et la certification linguistique reconnues internationalement sont un atout pour les élèves portugais - il y a donc une valorisation académique, professionnelle, personnelle et sociale.

Deuxièmement, les épreuves sont organisées selon le Cadre Européen Commun de Référence pour les langues (CECR) et certifient le niveau de compétence linguistique acquis - d'A1 à B2.

Finalement, il faut remarquer qu'un groupe de travail a été créé en 2014, en incluant représentants du MEC et de l'IFP, dans le but d'établir le cadre institutionnel et juridique de ce processus au Portugal.

\section{Information additionnelle}

On peut trouver de l'information sur le système éducatif portugais, la législation, les documents pédagogiques de référence, les plans d'études et les projets nationaux sur le site de la Direction générale de l'Éducation : http://www.dge.mec.pt.

Il y a aussi de l'information disponible sur le système éducatif portugais sur le site d'Eurydice : https://webgate.ec.europa.eu/fpfis/mwikis/eurydice/index.php/ Portugal :Overview

Pour des informations à propos des programmes et des projets développés dans le domaine $d u$ français, vous pouvez contacter directement la DGE, par courrier électronique (eulalia.alexandre[at]dge.mec.pt et ana.mayer[at]dge.mec.pt).

\section{RÉSUMÉS}

Les objectifs de cette communication ont été de présenter le système éducatif portugais, ses principes et priorités, sa structure, ses cours, le curriculum de l'enseignement élémentaire et celui de l'enseignement secondaire, ainsi que l'évaluation et la certification; d'analyser la 
situation particulière de l'enseignement du français au Portugal, soit à l'enseignement élémentaire, soit à l'enseignement secondaire, dans le cadre des langues vivantes étrangères et des dernières données statistiques; et de montrer, dans le cadre du Protocole de Coopération Éducative franco-portugais du 10 avril 2006, les différents programmes et projets innovants qui sont actuellement développés au Portugal, tels que les Assistants de Français, les Sections Européennes de Langue Française, les Séjours Professionnels et la certification DELF scolaire.

This communication aimed at presenting the Portuguese education system, its principles and priorities, its structure, its courses, the primary education curriculum and that of secondary education, as well as assessment and certification; analysing the specific situation of the teaching of French in Portugal, either in primary education or in secondary education, in the context of foreign languages and the latest statistics; and showing, in the framework of the FrenchPortuguese Protocol on Educational Cooperation of 10th April 2006, various innovative programmes and projects that are being developed in Portugal, such as the French Assistants, the French European Sections, the Professional Stays and the DELF Scolaire certification.

\section{INDEX}

Mots-clés : système éducatif, enseignement élémentaire, enseignement secondaire, enseignement du français, programmes et projets

Keywords : education system, primary education, secondary education, teaching of French, programmes and projects

\section{AUTEUR}

\section{EULÁLIA ALEXANDRE}

Direção-Geral da Educação

eulalia.alexandre[at]dge.mec.pt 